\title{
A mathematical modelling tool for unravelling the antibody-mediated effects on CTLA-4 interactions
}

\author{
Aravindhan Ganesan ${ }^{1 \dagger}$, Theinmozhi Arulraj ${ }^{1,2+}$, Tahir Choulli ${ }^{3}$ and Khaled H. Barakat ${ }^{1,4^{*}}$ (D)
}

\begin{abstract}
Background: Monoclonal antibodies blocking the Cytotoxic T-lymphocyte antigen 4 (CTLA-4) receptor have revolutionized the field of anti-cancer therapy for the last few years. The human T-cell-based immune responses are modulated by two contradicting signals. CTLA-4 provides a T cell inhibitory signal through its interaction with B7 ligands (B7-1 and B7-2), while CD28 provides a stimulatory signal when interacting with the same ligands. A previous theoretical model has focused on understanding the processes of costimulatory and inhibitory complex formations at the synapse. Nevertheless, the effects of monoclonal antibody (mAb)-mediation on these complexes are relatively unexplored. In this work, we expand on the previous model to develop a new mathematical framework for studying the effects of anti-CTLA-4 mAbs on the co-stimulatory (CD28/B7 ligands) and the Coinhibitory (CTLA-4/B7 ligands) complex formation at the immunological synapse. In particular, we focus on two promising anti-CTLA-4 mAbs, tremelimumab (from AstraZeneca) and ipilimumab (from Bristol-Myers Squibb), which are currently in clinical trials and the market, respectively, for targeting multiple tumors.
\end{abstract}

Methods: The mathematical model in this work has been constructed based on ordinary differential equations and available experimental binding kinetics data for the anti-CTLA-4 antibodies from literature.

Results: The numerical simulations from the current model are in agreement with a number of experimental data. Especially, the dose-curves for blocking the B7 ligand binding to CTLA-4 by ipilimumab are comparable with the results from a previous competitive binding assay by flow cytometry and ELISA. Our simulations predict the dose response and the relative efficacies of the two mAbs in blocking the inhibitory CTLA-4/B7 complexes.

Conclusions: The results show that different factors, such as multivalent interactions, mobility of molecules and competition effects, could impact the effects of antibody-mediation. The results, in particular, describe that the competitive effects could impact the dose-dependent inhibition by the mAbs very significantly. We present this model as a useful tool that can easily be translated to study the effects of any anti-CTLA-4 antibodies on immunological synaptic complex formation, provided reliable biophysical data for mAbs are available.

Keywords: CTLA-4, Immune checkpoints, Ipilimumab, Tremelimumab, Antibody, Mathematical modeling

\footnotetext{
* Correspondence: kbarakat@ualberta.ca

${ }^{\dagger}$ Aravindhan Ganesan and Theinmozhi Arulraj contributed equally to this work.

${ }^{\dagger}$ Equal contributors

${ }^{1}$ Faculty of Pharmacy and Pharmaceutical Sciences, University of Alberta, Edmonton, Canada

${ }^{4}$ Li Ka Shing Institute of Virology, University of Alberta, Edmonton, Canada

Full list of author information is available at the end of the article
}

(c) The Author(s). 2018 Open Access This article is distributed under the terms of the Creative Commons Attribution 4.0 International License (http://creativecommons.org/licenses/by/4.0/), which permits unrestricted use, distribution, and reproduction in any medium, provided you give appropriate credit to the original author(s) and the source, provide a link to the Creative Commons license, and indicate if changes were made. The Creative Commons Public Domain Dedication waiver (http://creativecommons.org/publicdomain/zero/1.0/) applies to the data made available in this article, unless otherwise stated. 


\section{Background}

Blockade of immune checkpoints has recently been proven as a revolutionary strategy in the fight against cancers [1-6]. T-cells play a pivotal role in modulating the immune response against pathogens [7] and also in the prevention of autoimmunity [6]. According to the classic 'two-signal' model in immunology [8, 9], T-cellmediated immune responses are activated by two different protein-protein interactions taking place at the immunological synapse $[1-5,10-12]$ (Fig. 1). The first signal is triggered when the $\mathrm{T}$-cell receptors (TCRs) recognize the major histocompatibility complex (MHC) on the surface of antigen-presenting cells (APCs). A second signal comes from the binding of CD28, a coreceptor expressed on the T-cell surface, with its' complementary B7 ligands, known as B7-1 (or CD80) and B7-2 (or CD86), on the surface of APCs. This second signal is required to activate and sustain the activity of T-cells [3, 5, 13, 14]; hence it is known as a costimulatory signal and $\mathrm{CD} 28$ is dubbed as a costimulatory receptor (Fig. 1). On the other hand, there are a number of negative signal stimuli (known as the inhibitory receptors) that act to inactivate the T-cells, including cytotoxic T-lymphocyte-associated protein-4 (CTLA-4 or CD152) and programmed death 1 (PD1 or CD279) [3]. Particularly, CTLA-4, a CD28 homologue (with $\sim 30 \%$ sequence identity) expressed on the T-cell surface, binds with the same set of B7-ligands of CD28; albeit with higher affinity towards B7-1 and
B7-2. Both CD28 and CTLA-4 are transmembrane proteins of the immunoglobulin superfamily that exist as homodimers [15] .Nevertheless, CD28 is known to bind the ligands monovalently; whereas, CTLA-4 is bivalent in nature that, infact, allows it to bind the B7 ligands with high avidity $[15,16]$. Given such differences, under competitive environments, CTLA-4 would be able to out-compete CD28 for ligand-binding [17]. The functionalities of CD28 and CTLA-4 are interlinked and both co-stimulatory and co-inhibitory signals are required for maintaining the immunological balance between self-tolerance and defending against foreign entities and pathogens under normal conditions $[4-6,18,19]$.

Cancer cells are able to escape the immunological surveillance of T-cells, by overexpressing the inhibitory receptors that attenuate anti-tumor immune response $[4,5]$. As a result, in principal, blocking the co-inhibitory receptor-ligand interactions (schematically shown in Fig. 1) should be able to facilitate the re-activation of T-cells, which in-turn will recognize and eliminate cancers. Monoclonal antibodies (mAbs) blocking inhibitory immune checkpoints have demonstrated exceptional therapeutic benefits in clinical trials [2022], which is transforming human cancer treatment. Ipilimumab, a completely human IgG1 antibody from Bristol-Myers Squibb, became the first-in-class antiCTLA-4 mAb to be approved by the US Food and Drug Administration (FDA) in 2011 for the treatment of metastatic melanoma [23, 24]. Several clinical trials showed

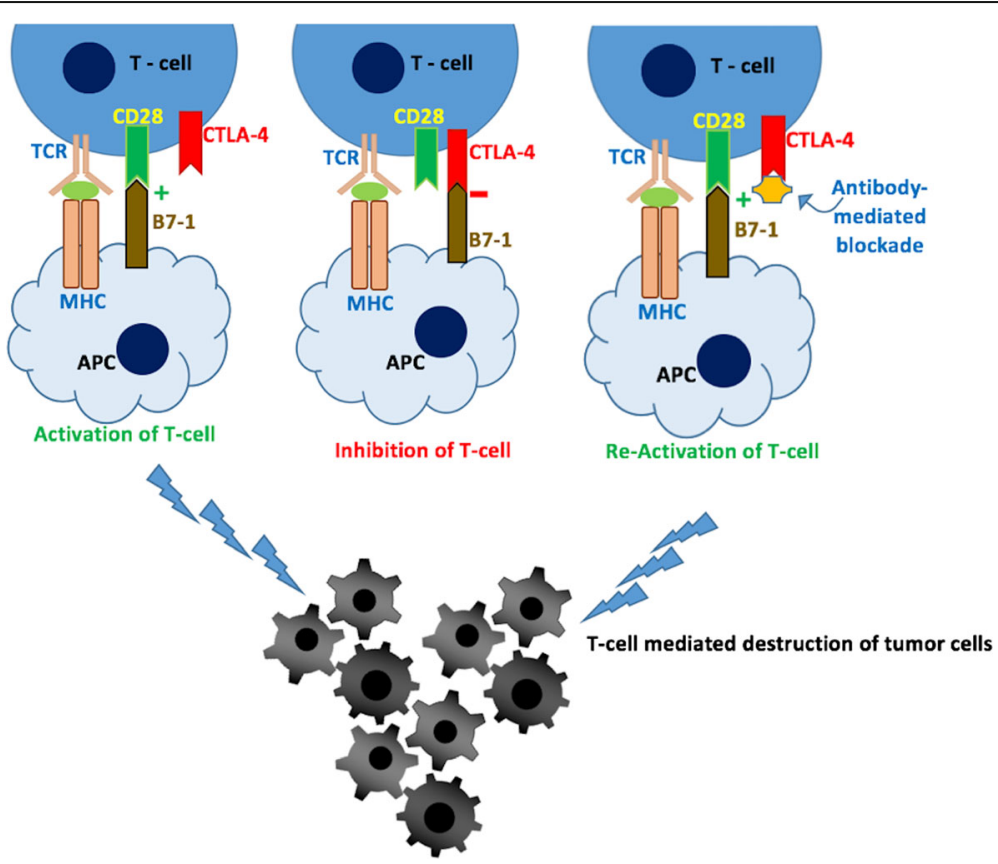

Fig. 1 Schematic representation of T-cell activation and inhibition by the interactions of CD28 and CTLA-4 with the B7 ligands, B7-1 and B7-2, respectively 
that monotherapy with ipilimumab in metastasis melanoma patients increased the overall responsive rate by $10-20 \%[1,25]$. When combined with other immune checkpoint therapies, ipilimumab is able to offer much enhanced benefits [26, 27]. Another IgG2 antiCTLA-4 mAb, Tremelimumab from AstraZeneca, is currently in phase III clinical trials $[1,5]$. Similar to Ipilimumab, AstraZeneca's tremelimumab binds specifically to CTLA-4 and blocks its interaction with the B7 ligands. Tremelimumab is also being tested in combination with other immune checkpoint mAbs for targeting multiple tumors $[28,29]$, including melanoma, colon cancer, and mesothelioma [28, 29]. The progress made by these two antibodies, from bench to final clinical trial phases or to the market, has boosted the interests towards developing more promising immune-checkpoint blocking inhibitors (both mAbs and, lately, small molecules).

Very recently, $\mathrm{He}$ et al. [1] investigated the binding profiles of both mAbs against CTLA-4 using surface plasmon resonance (SPR) experiments and reported their $K_{D}$ values as $18.2 \mathrm{nM}$ for ipilimumab/CTLA-4 complex and $5.89 \mathrm{nM}$ for tremelimumab/CTLA-4 complex. These binding affinity values are higher than (or, in some cases, comparable to) those reported for B7-1 ligands with CTLA-4 [1]. However, it is important to note that different range of affinity values have been reported for the CTLA-4/B7-1 complexes. For example, an earlier study reported that a soluble B7-1 Ig fusion protein bound to CTLA-4 with a dissociation constant of $\sim 12 \mathrm{nM}$ [30]. However, this value was argued to be higher when compared with other protein-protein interactions occurring between T-cell surface and APCs [31]. The authors of this work [30] had described that the CTLA-4Ig in their experiments was not monomeric in solution, and possibly formed higher aggregates that might have possibly resulted in the high apparent $K_{D}$ values for the interactions of CTLA-4Ig/B7Ig fusion protein. Another study [31] based on SPR experiments reported that, at $37{ }^{\circ} \mathrm{C}$, soluble recombinant B7-1 bound to CTLA-4 with a $K_{D}$ value of $0.42 \mu \mathrm{M}$. This indicates that affinity between B7-1 and CTLA-4 is relatively lower than that of ipilimumab/CTLA-4 and tremelimumab/CTLA-4 complexes. The association rate constant $\left(\mathrm{k}_{\mathrm{a}}\right)$ of ipilimu$\mathrm{mab}\left(3.83 \times 10^{5} / \mathrm{Ms}\right)$ and tremelimumab $\left(3.08 \times 10^{5} / \mathrm{Ms}\right)$ are almost close to each other, however, their dissociation rate constants $\left(k_{d}\right)$ were significantly different [1]. The $\mathrm{k}_{\mathrm{d}}$ value for ipilimumab was $6.96 \times 10^{-3} / \mathrm{s}$, whereas for tremelimumab it was $1.8 \times 10^{-3} / \mathrm{s}$ [1]. This clearly indicates that the tremelimumab is able to form much stable complex with CTLA-4 when compared to that of ipilimumab. Thus, understanding the effects of these two potent anti-CTLA-4 antibodies on the co-stimulatory and the co-inhibitory complex formation at the synapse will be useful to develop effective next-generation anti-CTLA-4 therapeutics. The ultimate objective of this work is to precisely study these effects using mathematical modelling and simulations.

Mathematical modelling and simulation remains a powerful tool to gain quantitative insights into the dynamics of complicated systems [32-40]. Especially, mathematical modelling is gaining more popularity in the field of cancer immunotherapy. For example, Kirschner et al. [38] developed a mathematical model, based on ordinary differential equations (ODEs) to simulate the dynamics between tumor cells, immune-effector cells and cytokine interleukin-2. This model was useful to explore the effects of adoptive cellular immunotherapy (ACI) on the model and found that a combination therapy with ACI and IL-2 could boost the immune system sufficiently to clear the tumor [38]. Krnoik et al. [37] developed an expanded mathematical model, from a previously published model, in order to simulate cellular immunotherapy in melanoma. This model was useful to understand the findings from clinical trials suggesting that patients with the smallest tumor load respond better for this kind treatment [37]. Mathematical models have also been useful to understand tumor growth, response to therapy and the interactions of immune cells with the cancer cell [39-42]. Bidot et al. [43] developed a mathematical model for studying the kinetics of monoclonal T-cell-specific activation. This model attempted to account for the sequence of events starting from the TCR-MHC binding to T-cell activation and response [43]. Mathematical modelling has also been employed to study the complex formation between PD-1 receptor and its ligands [44]. Jansson et al. [33] developed a theoretical model for simulating the synaptic accumulation of the molecules involved in co-stimulation and inhibition of T-cells. This model, which was developed based on the Ordinary Differential Equations (ODEs) and rigorous biophysical and expression data from literature, explained the interactions of CTLA-4 and CD-28 with their B7ligands in the context of a potentially dynamic synaptic microenvironment [33]. However, until now, there is no model that could predict the response to CTLA-4 blocking antibodies and the consequent effects on complex formation at immunological synapse.

In this work, we expand the model of Jansson et al. [33] by including the effect of an anti-CTLA- 4 antibody. Our model is based on ODEs and additional biophysical data for antibody-CTLA-4 complexes [1]. Using this new model, we studied how the binding of mAbs (with different affinities) to CTLA-4 dynamically changed the interactions among co-inhibitory complexes (CTLA-4/B7-1 and CTLA-4/B7-2) and co-stimulatory complexes 
(CD28/B7-1 and CD28/B7-2) at the immunological synapse. The numerical simulations from the model have been validated by different experimental data reported earlier. This model should be a useful tool to predict the dose response of any anti-CTLA-4 antibodies and their impacts on synaptic complex formation processes.

\section{Methods}

\section{Mathematical modelling assumptions}

As mentioned above, the mathematical model in this work is based on the previous two-compartment model by Jansson et al. [33], which was constructed to simulate the CD28 and CTLA-4 complex formation with the B7ligands at immunological synapses. In this study, we developed an expanded model to assess the changes in the co-stimulatory (CD28/B7 ligands) and the co-inhibitory (CTLA-4/B7 ligands) interactions upon binding of the antibody to the free CTLA-4 receptor sites. This model, as in Jansson's model [33], involves two components that includes the synapse and the region outside of the synapse. It is assumed that CD28, B7-1 and B7-2 are primarily unbound and distributed uniformly over the surface. CTLA-4, on the other hand, is present intracellularly and gets injected into the synapse upon activation $[45,46]$. The 'free-diffusion' model has been applied to control the mobility of the molecules, such that only the mobile molecules are able to diffuse into the synapse, while the immobile species outside of the synapse are ignored. It is understandable that the immobile species outside of the synapse are, anyway, not able to participate in any complex formation. On the other hand, the immobile molecules inside the synapse stay there and are involved in the protein-protein interactions. The model also assumes that the CTLA- 4 receptors, once injected from the intracellular environment, stay within the synapse. In the case of antibody, unlike the membrane-bound ligands, it is in the free solution and is assumed to bind to free CTLA- 4 monovalently. The model assumes that the binding site of B7-1, B7-2 and the antibody molecules on CTLA- 4 overlap considerably [2], and, therefore, only one of them (B7-1 or B7-2 or the antibody) is able to bind with a CTLA-4 molecule. Moreover, in our model, the antibody is allowed to bind an unbound CTLA-4 monomer that is part of a dimer, where the other monomer can be bound with a B7 ligand. Due to the lack of parameters for the association and dissociation of complexes in the model, rate constants from similar complexes are used and reactions are modelled as parallel mass action reactions. Bivalent association and dissociation rate constants are employed for the binding/unbinding of B7 ligands to a CTLA-4 monomer that is part of a dimer, where the other monomer is bound to an antibody.

\section{Parameters}

The parameters for the interactions of CTLA-4 and CD28 with the B7 ligands in the current model are almost similar to those in Jansson's model [33], which are given in the supplementary information (Additional file 1: Table S1). However, the association and dissociation rate constants for the interactions of antibodies, tremelimumab and ipilimumab, with CTLA-4 receptor are collected from the literature [1] and employed in the current model. The reported SPR experiments [1] measured the association rate constants of ipilimumab and tremelimumab with CTLA- 4 to be $3.83 \times 10^{5} / \mathrm{Ms}$. and $3.08 \times 10^{5} / \mathrm{Ms}$., respectively. Whereas, their dissociation rate constants were observed to be 6 . $96 \times 10^{-3} / \mathrm{s} \quad$ (ipilimumab) and $1.8 \times 10^{-3} / \mathrm{s}$ [1] (tremelimumab). It is important to note that the rate constants for the interactions of antibodies are not converted to 2-D rates, as the antibodies are present in the solution. This approximation is reasonable, when the binding site is accessible [47].

\section{Antibody complex formation}

The equations related to the modelling of rates of change for the complex formation between different species, such as CD28, CTLA-4, B7-1 and B7-2, are all similar to those of Jansson et al. [33]. However, a number of additional terms are employed in the current model in order to account for the increase or decrease in density of these species in response to the association and dissociation of antibodies to that of CTLA-4 monomers. Please note that the rate of change of the density of complexes for the newly added terms in the model are also written using the mass-action law.

\section{Rate of change of density of antibody/CTLA-4 complex}

The antibody associates with a CTLA-4 dimer at the rate of $\mathrm{k}_{\mathrm{on}}$, in order to form the antibody/(CTLA-4) complex (referred as Ac complex in the equation). The rate for dissociation of antibody from the Ac complex is $\mathrm{k}_{\text {off. }}$ An unbound B7-1 associates with the Ac complex at the rate of $\alpha_{44}$ to form a complex of Ac/B7-1 (or $E A b_{1}$ ), whereas, an unbound B7-2 associates with the Ac complex at the rate of $\alpha_{33}$ to form Ac/B7-2 (or Ab/CTLA4/B72). An antibody binds to the Ac complex at the rate of Kon to form the resultant $\mathrm{Ab} / \mathrm{Ac}$ (or AcA) complex. The association of either of the B7-1 ligands or antibody to the Ac complex reduces the density of the latter. On the other hand, dissociation of B7-1 $\left(\right.$ rate $\left.=\delta_{44}\right), B 7-2\left(\right.$ rate $\left.=\delta_{33}\right)$ and antibody $\left(\right.$ rate $\left.=k_{\text {off }}\right)$ from the EAb1, Ab/CTLA4/B72, AcA complexes, respectively, increases the density. Hence, the rate of change in the density of the Ac complex can be written as follows, 


$$
\begin{aligned}
\frac{d A c}{d t=}= & -\alpha_{44}{ }^{*} A c^{*} B 71+\delta_{44}{ }^{*} E A b_{1}-k_{o n}{ }^{*} A c^{*} A b+k_{o f f}{ }^{*} A c A \\
& +k_{o n}{ }^{*} C T L A 44^{*} A b-k_{o f f} A c-\alpha_{33}{ }^{*} A c^{*} B 72 \\
& +\delta_{33}{ }^{*} A b / C T L A 4 / B 72
\end{aligned}
$$

The rate of change of density for the other antibodymediated complexes are given in supplementary information, Additional file 2: Table S2. The numbers of free antibody molecules is increased by the dissociation of $\mathrm{mAb}$ from any of the antibody-included complexes (such as, Ac, AcA, Ab/CTLA-4/B72, EAb, CAb, DAb). On the other hand, association of free antibody to form any of the above complexes decreases the total number of free antibody molecules,and this rate of change in the antibody can be written as,

$$
\begin{aligned}
\frac{d A b}{d t}= & -k_{o n}{ }^{*} C T L A 4^{*} A b+k_{o f f}{ }^{*} A c-k_{o n}{ }^{*} A c^{*} A b \\
& +k_{\text {off }}{ }^{*} A c A-k_{o n}{ }^{*} A b^{*} \sum_{k=1}^{\infty} E_{k} \\
& +k_{o f f}{ }^{*} \sum_{k=1}^{\infty} E A b_{k}-k_{o n}{ }^{*} A b^{*} \sum_{k=1}^{\infty} C_{k} \\
& +k_{\text {off }} \sum_{k=1}^{\infty} C A b_{k}-k_{o n}{ }^{*} A b^{*} \sum_{k=1}^{\infty} C A b_{k} \\
& +k_{o f f}{ }^{*} \sum_{k=1}^{\infty} D A b_{k}-k_{o n}{ }^{*} C T L A 4 / B 72^{*} A b \\
& +k_{o f f}{ }^{*} A b / C T L A 4 / B 72
\end{aligned}
$$

Density of mobile CD28, B71 and B72 molecules, present outside synapse is estimated by subtracting the total number of mobile molecules of CD28, B71,B72 that are part of complexes formed at synapse.

$$
\begin{aligned}
C D 28 \text { out }= & t_{C D 28, \text { tot }}{ }^{*} m_{C D 28}-(C D 28+C D 28 / B 71 \\
& \left.+2^{*}(C D 28)_{2} / B 71+C D 28 / B 72-i_{C D 28}\right) * a_{s y n}
\end{aligned}
$$

$$
\begin{aligned}
\text { B71out }= & t_{B 71, \text { tot }}{ }^{*} m_{B 71}-\left(B 71+C D 28 / B 71+(C D 28)_{2} / B 71\right. \\
& +\sum_{k=1}^{\infty} B_{k}(k+1)+\sum_{k=1}^{\infty} C_{k} k+\sum_{k=1}^{\infty} E_{k} k \\
& +\sum_{k=1}^{\infty} E A b_{k} k+\sum_{k=1}^{\infty} C A b_{k} k \\
& \left.+\sum_{k=1}^{\infty} D A b_{k} k-i_{B 71}\right) * a_{s y n}
\end{aligned}
$$

$$
\begin{aligned}
\text { B72out }= & t_{B 72, \text { tot }}{ }^{*} m_{B 72}-(B 72+C D 28 / B 72+C T L A 4 / B 72 \\
& \left.+2^{*} C T L A 4 /(B 72)_{2}+A b / C T L A 4 / B 72-i_{B 72}\right) * a_{s y n}
\end{aligned}
$$

Similarly, the number of CTLA4 molecules present inside the cell is calculated by subtracting it from total number of CTLA4 molecules.

$$
\begin{aligned}
\text { CTLA4int }= & t_{\text {CTLA4,tot }}{ }^{*} m_{\text {CTLA4 }}-(C T L A 4+C T L A 4 / B 72 \\
& +C T L A 4 /(B 72)_{2}+\sum_{k=1}^{\infty} B_{k} k+\sum_{k=1}^{\infty} C_{k}(k+1) \\
& +\sum_{k=1}^{\infty} E_{k} k+\sum_{k=1}^{\infty} E A b_{k} k+\sum_{k=1}^{\infty} C A b_{k}(k+1) \\
& \left.+\sum_{k=1}^{\infty} D A b_{k}(k+1)+A b / C T L A 4 / B 72+A c+A c A\right) * a_{s y n}
\end{aligned}
$$

\section{Modelling and simulations}

Mathematical modelling and simulation procedures in this study were programmed using MATLAB software from MathWorks (https://www.mathworks.com/products/matlab.html). A stiff ordinary differential equation solver, ode15s, was used for solving the equations in the current model. The different components included in the model and their abbreviations are provided in the supplementary information (Additional file 3: Table S3). Each simulation was performed for $7 \mathrm{~h}$ (i.e., $25,200 \mathrm{~s}$ ) unless otherwise stated. Initially, the simulations are performed with ' 0 ' concentration of antibody, in order to reproduce the results from Jansson's model [33]. Later, the simulations were performed with different concentrations of tremelimumab and ipilimumab and the effects on CTLA-4 and CD28 complex formation were analyzed.

\section{Results}

Simulation of antibody-free complex formation

Initially, the simulation based on the free-diffusion model (Fig. 2a) was performed for $\sim 7 \mathrm{~h}$, without the inclusion of any antibody (i.e., $0 \mu \mathrm{M}$ antibody concentration).This situation is ideally to reproduce the simulation from Jansson et al. [33].This simulation mimics an activated T-cell environment, where both CD28 and CTLA-4 are present at the synapse and are competing for ligand-binding. During the initial hours ( $15 \mathrm{~min})$, the expression levels of B7-2 at synapse is much higher and hence, it remained the leading ligand for CTLA-4 and CD28. Particularly, at about $6 \mathrm{~min}$, the CTLA-4/B72 interactions reached a peak with $\sim 600$ CTLA-4 monomers were bound to B7-2, against 140 CTLA-4/B7-1 and $108 \mathrm{CD} 28 / \mathrm{B} 7-2$ complexes. The domination of CTLA-4/B7-2 remained until $\sim 17 \mathrm{~min}$, however, this number (of CTLA-4/B7-2 complex) dropped significantly to $<50$ at $\sim 1 \mathrm{~h}$ of simulation. Gradually, as more CTLA-4 moved from the intracellular region to the synapse (see in Fig. 2b), the CTLA-4/B7-1 complex replaced CTLA-4/B7-2 and became the most populated complex after $\sim 20 \mathrm{~min}$. After reaching the steady state, it can be seen (in Fig. 2a) that the majority of the B7-1 was bound to CTLA-4 (resulting 790 CTLA-4/B7-1 complexes), while only small numbers of B7-1 ( 50) 

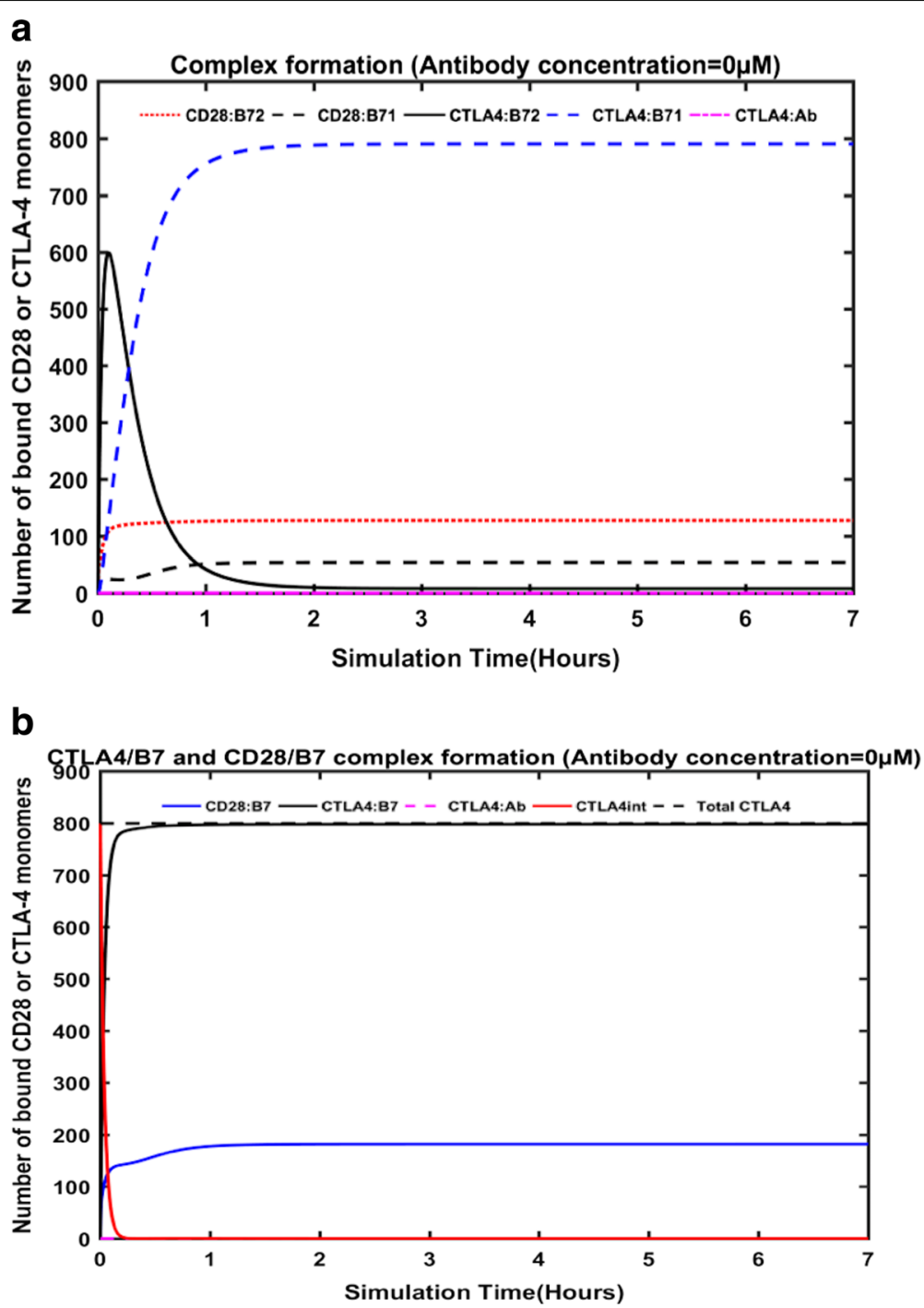

Fig. 2 Complex formation in the free-diffusion model from the antibody-free simulations. The number of CD28 and CTLA-4 molecules bound to B7-1 and B7-2 at the immunological synapse, during the antibody-free simulations (a). The dynamic changes in the total numbers of CTLA-4/B7 complex, CD28/B7 complex, amount of CTLA-4 at the intra-cellular environment and the total number of CTLA-4 monomers are provided (b)

were engaged with the $\mathrm{CD} 28$ receptor. On the other hand, the amount of CD28/B7-2 complexes $(\sim 127)$ outnumbers the complexes formed by CTLA- 4 monomers and B7-2( 8). As Jansson et al. [33] noted, these behaviors in the simulation are in good agreement with previous findings that $B 7-1$ is the preferred ligand for CTLA-4 and B7-2 preferentially recruits CD28 at the synapse $[48,49]$. Hence, $99 \%$ of CTLA- 4 are complexed with $B 7-1$, while only $1 \%$ of the receptor is engaged with the B7-2 ligand. Overall, however, the percentage of CTLA-4 complexed with the B7 ligands is much higher than that of CD28/B7 complexes (Fig. 2b). This trend is expected as CTLA-4 has a higher affinity towards B7 ligands than that of CD28.

\section{Binding of the antibodies to CTLA-4}

Initially, we performed two simulations with an in silico knockout model, where CD28 and the B7 ligands (both B7-1 and B7-2) were muted and only CTLA-4 was allowed to bind with the antibodies, ipilimumab and tremelimumab, at different concentrations $(0.002 \mu \mathrm{M}$ to $0.018 \mu \mathrm{M})$. These simulations were carried out in order to confirm that, in a non-competitive setting, $50 \%$ of CTLA- 4 had formed complex with the antibodies when the concentration of the antibodies equaled their respective $K_{D}$ values. As shown in Figs. 3, $50 \%$ of the CTLA- 4 monomers were bound to antibody at the concentrations of $0.018 \mu \mathrm{M}$ and $0.0058 \mu \mathrm{M}$ of ipilimumab (Fig. 3a) and tremelimumab (Fig. 3b), 

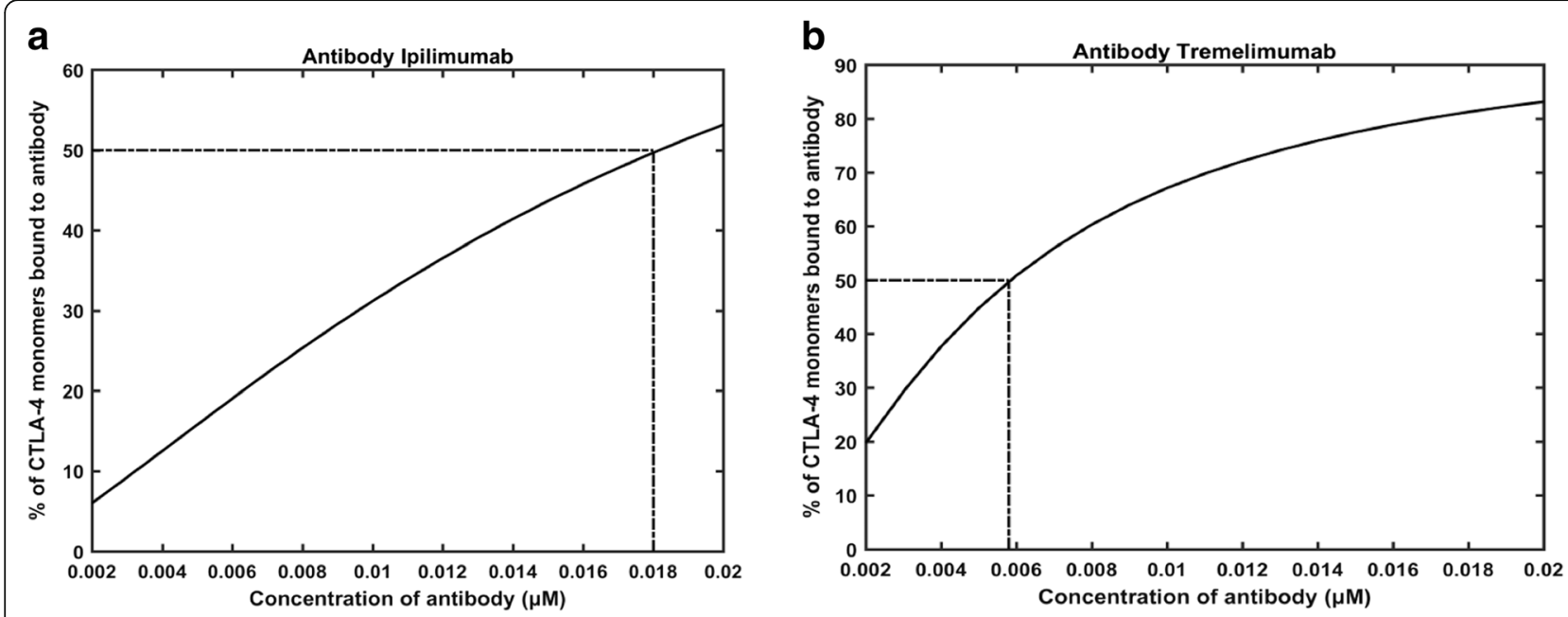

Fig. 3 The reproduction of the experimental $K_{D}$ values for the binding of CTLA-4 to ipilimumab (a) and tremelimumab (b)

respectively. These concentrations are approximately close to the $K_{D}$ values for these antibodies (ipilimumab $18.2 \mathrm{nM}$; Tremelimumab $-5.89 \mathrm{nM}$ ), as reported by $\mathrm{He}$ et al. [1] based on their SPR experiments. Nevertheless, since the association and dissociation rate constants of the antibodies for the current model was obtained from the work of $\mathrm{He}$ et al. [1], it is expected that the model is able to achieve $50 \%$ complex formation at the concentration of the $K_{D}$ values of mAbs. Hence, this confirms that the model simulates the complex formation correctly.

\section{Competitive binding of the antibodies to CTLA-4 and model validation}

Next, we performed the simulations to study how the antibodies, in the absence of CD-28, competed with either B7-1 or B7-2 for binding to CTLA-4. In order to perform these simulations, CD-28 and either of the B7 ligands were muted in the model. The resulting dose response curves for the percent inhibition of B7-1 binding and B7-2 binding with CTLA- 4 at different concentrations of the antibodies, as predicted by the model, are shown in Fig. 4a and b, respectively. As shown in Fig. 4a and b, the model was able to simulate the dose-dependent inhibition of CTLA4/B7 interactions by the competitive binding of ipilimumab and tremelimumab.

The dose-curves for blocking B7-1 and B7-2 binding by ipilimumab (Fig. 4a) is comparable with the experimental dose curves reported by Keler et al. [50] for the same antibody. It is important to note that no values from this experimental work have been used in our simulations or in the construction of our model. In this previous work [50], the scientists at Medarex performed competitive binding assay by flow cytometry and ELISA to demonstrate the ability of ipilimumab (dubbed as 10D1 in the paper) [50] to block the interactions of CTLA-4 with B7-1 and B7-2, separately. The simulations based on our model was also carried out by having only one of the B7 ligands active at a time, so as to mimic the experimental set-up [50]. Although the overall trends in the dose-response curves for ipilimumab obtained from our model and the previous experiment [50] are in agreement, the percent inhibition predicted for each dose of the antibody by our model are higher than those observed in the experiments [50]. For example, our model predicts that, at $10 \mu \mathrm{M}$ concentration of ipilimumab, $\sim 89 \%$ of $B 7-1$ and $92 \%$ of B7-2 are blocked; the experiments, on the other hand, reported $\sim 70 \%$ and $\sim 90 \%$ inhibition of B7-1 and B7-2 binding [50], respectively. Nevertheless, it should be noted that the previous experiments [50] were carried out with a human CTLA-4 (hCTLA-4) expressing cell, which was constructed by a hCTLA-4/murineCD3 chimeric gene. Thus, taking into account this difference and other experimental conditions, in general, the predictions from our model and the experiments are in reasonable agreement. Particularly, Keler et al. [50] reported an $\mathrm{IC}_{50}$ value of $\sim 1-3 \mu \mathrm{M}$ for ipilimumab (or 10D1 as named in the paper) to block the B7 ligands, which is in excellent agreement with the values predicted by our model $\left(\mathrm{IC}_{50}=1.11 \mu \mathrm{M}\right.$ for B7-2 blocking; and $3.5 \mu \mathrm{M}$ for B7-1 blocking). This validates the ability of our model to simulate the competitive binding between antibodies and the B7 ligands reasonably well.

By comparing the dose-response curves for the two antibodies as predicted from our simulations, it is 


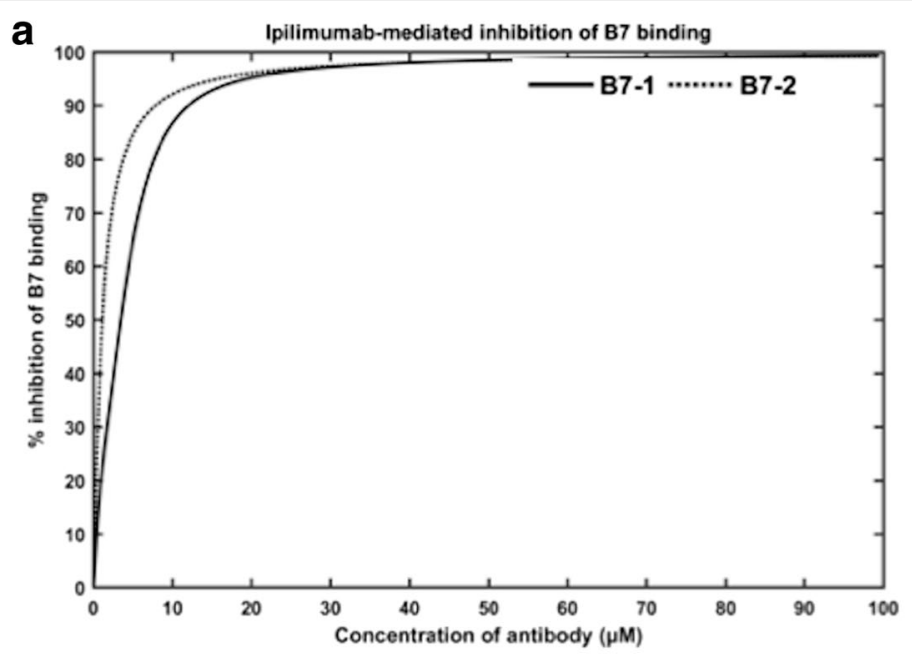

b Tremelimumab-mediated inhibition of B7 binding

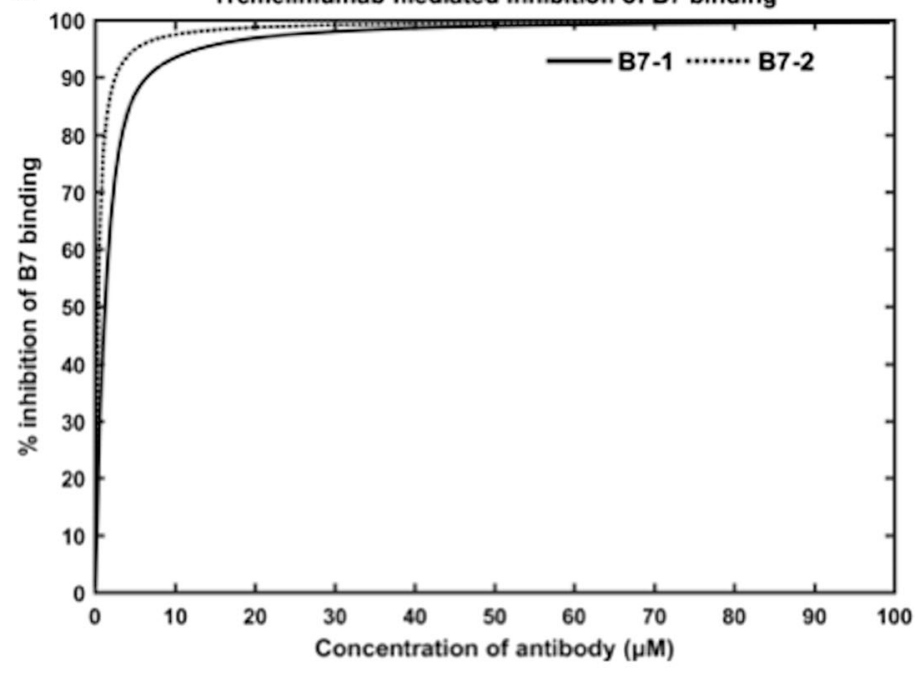

C CTLA4:B72 - - CTLA4:B71

C

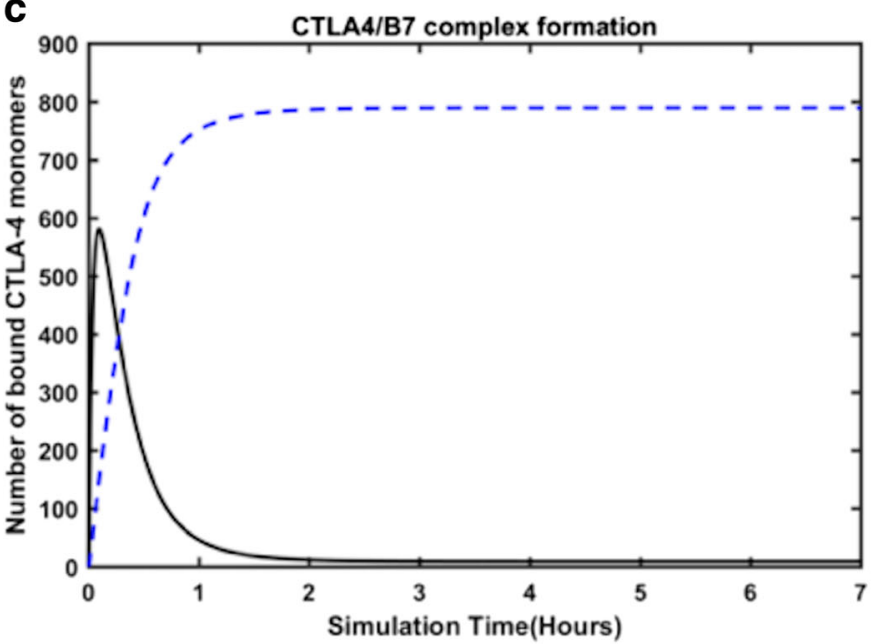

Fig. 4 The dose-curves for the blocking of B7 ligands to CTLA-4 by ipilimumab (a) and tremelimumab (b) in the simulations performed in either of the B7 -knock-out model, where either of the B7 ligands and CD28 were removed. The complex formation of CTLA-4/B7-1 and CTLA-4/B7-2 in the absence of CD28 and antibody is also simulated (c) 
apparent that, at any given dose concentration, tremelimumab (Fig. 4b) is able to inhibit higher percentage of B7-1 and B7-2, when compared to that of ipilimumab (Fig. 4a). For instance, $1 \mu \mathrm{M}$ of tremelimumab was found to inhibit $\sim 54 \%$ of B7-1 binding and $\sim 76 \%$ of B7-2 binding, which are much higher \% inhibition than those obtained from $1 \mu \mathrm{M}$ of ipilimumab ( $25 \%$ of B7-1 inhibition; $\sim 47 \%$ inhibition of B7-2) in our simulations. Such trends are in line with the affinity of these antibodies against CTLA-4 as reported by $\mathrm{He}$ et al [1]. It has been reported that both the antibodies have comparable association rate constants, however, the dissociation rate constants of ipilimumab is much higher than that of tremelimumab [1]. Hence, tremelimumab is able to block much higher amounts of B7 ligands from binding to CTLA4 , when matched with ipilimumab. In addition, it can be noted that higher percent inhibition of CTLA-4/ B7-2 interactions than CTLA-4/B71 interactions can be achieved with low concentrations $(\leq 10 \mu \mathrm{M})$ of the antibodies. This again accords with previous observation that the affinity of CTLA-4 to B7-1 is higher than its affinity to B7-2 [51]. In order to test this statement, we performed a simulation with our model, where only $\mathrm{B} 7-1$ and $\mathrm{B} 7-2$ were allowed to competitively bind with CTLA-4. To model this scenario, we again left the total initial concentrations of the antibodies and the CD28 receptor to 0 , such that they do not have any effects on CTLA-4 binding to the B7 ligands. The result from this simulation is shown in Fig. 4c. Except for the first few minutes of the simulations, when the synapse was dominated by B7-2, the predominant amount of CTLA-4 remained in complex with B7-1 and the proportion of CTLA4/B7-2 complex was meager.

\section{Effects of antibody-mediation on the overall complex formation}

Subsequently, we tested the effects of antibodymediation on the co-stimulatory (CD28/B7 ligands) and the co-inhibitory (CTLA-4/B7 ligands) complex formation at the synapse. Simulations were performed with a restraint-free competitive environment facilitated by the model, where all the species (such as CTLA-4, B7-1, B7-2 and CD28) were present and a specific concentration of either of the antibodies was added. Initially, $10 \mu \mathrm{M}$ concentration of ipilimumab (Fig. 5a) and tremelimumab (Fig. 5b) were added in the simulations to study their effects. The results are comparable to Fig. 2a, where the simulation performed without the antibodies (concentration $=0 \mu \mathrm{M}$ ) is provided. As expected, upon addition of $10 \mu \mathrm{M}$ concentration of antibodies, the antibody-bound CTLA-4 complex out-numbered the other complex formations. Particularly, in the antibody-free simulations, the B7-2-bound CTLA-4 complex $(\sim 600)$ was dominant during the initial hours; however, the presence of the antibody reduced the initial dominance of this complex (CTLA-4/B7-2) by at least $75 \%$.

In the case of simulation with ipilimumab (concentration $=10 \mu \mathrm{M})$, it can be seen that both the B7 ligands and ipilimumab contested for CTLA-4 binding during the early hours. However, the antibody outcompeted the B7 ligands to form complexes with CTLA-4. It can be seen in Fig. 5a that, at the end of the simulations, there were $\sim 630$ CTLA-4/ipilimumab complexes against $\sim 129$ CTLA-4/B7-1 complexes. This is, particularly, a significant reduction in the sheer dominance of CTLA-4/B7-1 complexes (790 numbers) seen in the antibody-free simulations. On the other hand, $10 \mu \mathrm{M}$ concentration of tremelimumab was able to bind more effectively with CTLA-4, thus remaining the most populated complex $(\sim 760$ CTLA-4/tremelimumab complexes) at the steady state of the simulations, as shown in Fig. 5b. As a result, there were only 20 complexes of CTLA-4 and B7-1 at the end of simulations with tremelimumab $(10 \mu \mathrm{M})$. In summary, the antibody-mediation has significantly impacted the CTLA-4/B7 ligand complex formation, which reduced from $\sim 800$ numbers (in antibody-free simulations) to $\sim 168$ and $\sim 42$ in the simulations with $10 \mu \mathrm{M}$ of ipilimumab and tremelimumab, respectively. Later, we increased the dose of the antibodies to $15 \mu \mathrm{M}$ and performed simulations (Fig. 5c-d). The higher dose of antibodies naturally increased the effects on CTLA-4 binding and B7-ligand blockade. In fact, predominant CTLA-4 monomers were engaged in complexes with the antibodies, ipilimumab $(\sim 707)$ and tremelimumab $(\sim 780)$. And as a result, the inhibitory complex formation between CTLA-4 and the B7-ligands had significantly diminished ( 23 with $15 \mu \mathrm{M}$ of tremelimumab; $\sim 92$ with $15 \mu \mathrm{M}$ of ipilimumab). However, the addition of antibody did not significantly impact the interactions of CD28 with the B7 ligands. While the amount of CD28/B7-2 complexes remained almost the same in antibody-free and antibody-included simulations, the number of CD28/B71 complexes increased only slightly. The total numbers of different complexes (such as CTLA-4/mAb, CTLA-4/B7 and CD28/B7) at the end of simulations performed with different concentrations of ipilimumab and tremelimumab are summarized in Table 1.

Next, we simulated full dose-response curves for the two antibodies (Fig. 6), ipilimumab and tremelimumab. The inhibition percentage of the $\mathrm{B} 7$ ligands (i.e., B7-1 and B7-2) at each concentration of the antibodies in this figure was calculated as follows, 


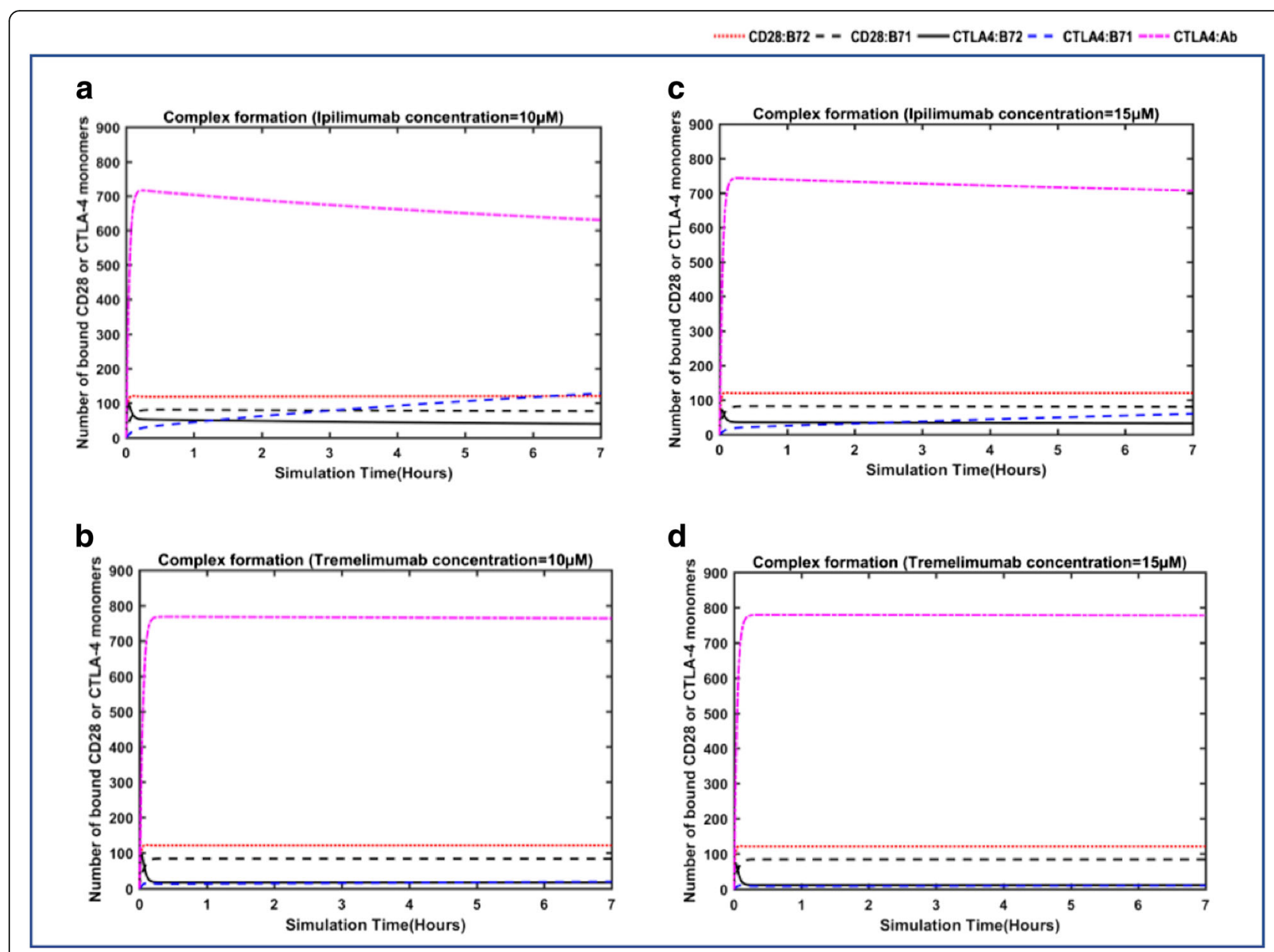

Fig. 5 Antibody-mediated changes in the complex formation at the synapse in the free-diffusion model: The dynamic changes in the different complexes, such as CTLA-4/antibody, CTLA-4/B7 ligands and CD28/B7 ligands, at the synapse are simulated at $10 \mu \mathrm{M}$ and $15 \mu \mathrm{M}$ of antibodies, ipilimumab (a, c) and tremelimumab (b, d)

$\%$ B7 inibition@x $\mu M$ of $m A b$

$$
=\left(1-\frac{(\text { No.of CTLA-4/B7 complex }) @ x \mu M \text { of } m A b}{(\text { No.of CTLA-4/B7 complex }) @ 0 \mu M \text { of } m A b}\right) \times 100
$$

Table 1 Comparison of the total numbers of CTLA-4/mAb, CTLA-4/B7 and CD28/B7 complexes formed at the end of simulations performed with the different concentrations $(=0 \mu \mathrm{M}, 10 \mu \mathrm{M}$ and $15 \mu \mathrm{M})$ of ipilimumab and tremelimumab

\begin{tabular}{|c|c|c|c|c|c|c|}
\hline \multirow[t]{3}{*}{ Interactions } & \multicolumn{6}{|c|}{ Number of interacting CTLA-4/CD28 monomers } \\
\hline & \multirow[t]{2}{*}{ Initial } & \multirow{2}{*}{$\begin{array}{l}\text { Antibody } \\
=0 \mu \mathrm{M}\end{array}$} & \multicolumn{2}{|c|}{ Tremelimumab } & \multicolumn{2}{|c|}{ Ipilimumab } \\
\hline & & & $(10 \mu \mathrm{M})$ & $(15 \mu \mathrm{M})$ & $(10 \mu \mathrm{M})$ & $(15 \mu \mathrm{M})$ \\
\hline CD28/B7-2 & 0 & 127 & 121 & 121 & 121 & 120 \\
\hline CD28/B7-1 & 0 & 54 & 83 & 84 & 77 & 81 \\
\hline CTLA-4/B7-2 & 0 & 8 & 17 & 12 & 40 & 32 \\
\hline CTLA-4/B7-1 & 0 & 790 & 20 & 11 & 129 & 60 \\
\hline CTLA-4/mAb & 0 & 0 & 763 & 778 & 631 & 707 \\
\hline
\end{tabular}

From the dose curves, it is clear that both the antibodies have effectively inhibited the bivalent interactions of CTLA-4 and B7-1 even at very small concentrations. In fact, $50 \%$ of the B7-1 interactions with CTLA-4 were inhibited at concentrations $<5 \mu \mathrm{M}$ in the case of both the antibodies. As much as $10 \mu \mathrm{M}$ of either of the antibodies was sufficient to achieve $\sim 90 \%$ (or more) inhibition of CTLA-4/B7-1 interactions (Fig. 6a). These percentage inhibition values for CTLA-4/B7-1 interactions shown by the antibodies in the fully competitive simulations are almost similar to those observed for B72 knockout-simulations (where B7-2 and CD28 were absent, in Fig. 4). Nevertheless, the inhibition of B7-2 interactions (with CTLA-4), in the full model simulations, required really very high dose of the antibodies. In fact, it can be seen that the CTLA-4/B7-2 inhibition was seen only at concentrations $\geq 20 \mu \mathrm{M}$ for Tremelimumab and $>60 \mu \mathrm{M}$ for ipilimumab (Fig. 6b). This indicates that at lower concentrations of the antibodies, 


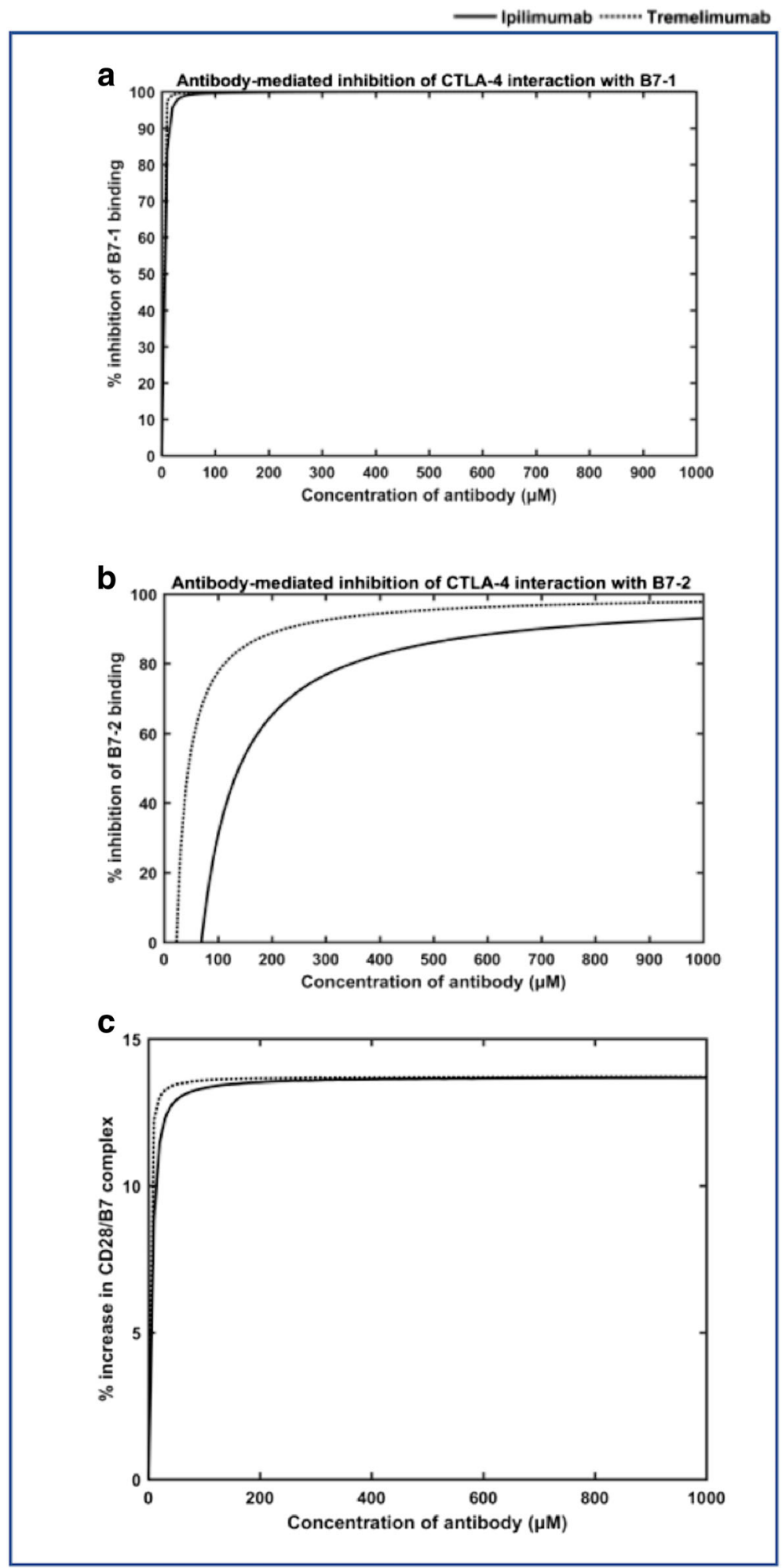

Fig. 6 The simulation of full dose-response curves for the two antibodies, ipilimumab (a) and tremelimumab (b) in the free-diffusion model. The $\%$ increase in the CD28/B7 complex during the full-dose response simulations with ipilimumab and tremelimumab are also plotted (c) 
there were actually some increase in the CTLA-4/B7-2 complexes, when compared to those seen in the untreated (or antibody-free) simulations. It should be noted that, in the antibody-free simulations, the CTLA-4/B7-2 complex dominated during the initial stages until the bivalent CTLA-4/B7-1 complex suppressed the monovalent CTLA-4/B7-2 interactions to become the dominant complex (see in Fig. 2). Whereas, in the antibody-mediated simulations, at low dose concentrations, the antibodies are more proactive in blocking the multivalent CTLA-4/B7-1 interactions, which relieves the suppression on the monovalent CTLA-4/B7-2 interactions. In addition, the actual numbers of CTLA-4/B72 complexes are in general much less than that of CTLA-4/B7-1 complex. For example, at the end of antibody-free simulations, there were only 8 CTLA-4/B7-2 complexes, when compared to 790 CTLA-4/B7-1 complexes. Nevertheless, higher antibody concentrations effectively blocked the CTLA-4/B7-2 interactions as well. This contradicts with the B7-1 knockout simulations (Fig. 4), where even the lower amounts of the antibodies inhibited predominant CTLA-4/B7-2 interactions. This suggests that the competitive effects could implicate the dose response predictions significantly. Finally, as indicated earlier, inhibition of CTLA-4/B7 interactions did not essentially translate to the more proportional increase in the $\mathrm{CD} 28 / \mathrm{B} 7$ complex formation. Figure $6 \mathrm{c}$ compares the percentage of increase in the CD28/B7 complex seen at each dose concentration (from $0 \mu \mathrm{M}$ to $1000 \mu \mathrm{M}$ ) of ipilimumab and tremelimumab. It can be seen that there were only a maximum of $\sim 14 \%$ gain in the CD28/B7 complexes following the inhibition of CTLA-4/B7 interactions by the mAbs in this study.

\section{Effects of antibody-mediation on the overall complex formation}

It is important to acknowledge that any mathematical model is mainly dependent on the parameters employed to build it. Particularly, the sensitivity is much higher in the models that rely on biological parameters. For example, Jansson et al. [33] tested the dependence of their model on the affinity, mobility and expression levels of various species, by reducing each of these parameters by 10 -fold, and found that some of the simulated interactions are sensitive to these changes [33]. However, it is true even in the case of measured data, as the experimental conditions, such as stoichiometry and temperature, have been shown to affect the results. For instance, an earlier experimental study reported that the affinity between CTLA-4 and B7-1 was 12 nM [30]; while another experimental study reported the affinity for the same complex to be $0.4 \mu \mathrm{M}$ [31]. In the current study, we tested the sensitivity of our model towards the changes in the rate constants for association and dissociation of different species in the model (the parameters, P2-P20 listed in Additional file 1: Table S1). To achieve this, we perturbed the values for each of these parameters in the range of -50 to $+50 \%$ from their respective original values used in the model. Figure 7 compares the effects of changing the association $\left(\mathrm{k}_{\text {on }}\right)$ and dissociation $\left(\mathrm{k}_{\text {off }}\right)$ rate constants for tremelimumab on different interactions, such as CTLA-4/antibody complex (Fig. 7a), CTLA-4/B7-1 (Fig. 7b), CTLA4/B7-2 (Fig. 7c), CD28/B7-1 (Fig. 7d) and CD28/B7-2 (Fig. 7e). As expected, the CTLA-4/antibody and the CTLA-4/B7 interactions were the most affected by these perturbations; indeed, the effects seen for CTLA-4/antibody complex were inverse to those of CTLA-4/B7 interactions. For example, the 50 -fold reduction in the $\mathrm{k}_{\text {on }}$ value led to the drop of $\sim 60$ numbers of monomer CTLA-4/antibody complexes, which was compensated by the increase in the total numbers of CTLA-4/B7 complexes (approximately +48 for CTLA-4/B7-1 complex; + 12 for CTLA-4/B7-2 complex). On the other hand, 50-fold increase in the $k_{\text {on }}$ value resulted in a small gain of CTLA-4/antibody complexes, which again led to the drop in the total numbers of CTLA-4/B7 complexes (refer to Fig. 7a-c). Similar inverse effects in the CTLA-4/antibody and CTLA-4/B7 complexes were also seen for the changes in the $k_{\text {off }}$ values. Nevertheless, the CD28/B7 interactions were mostly insensitive to these perturbations. This highlights the fact that CD28 is not able to compete with the high-affinity and highavidity interactions of CTLA-4/B7 and CTLA-4/antibody interactions.

The effects of varying the rate constants for association and dissociations for monovalent CD28/B7 complexes, monovalent CTLA-4/B7 complexes, bivalent CD28/B7-1 complex, and multivalent CTLA-4/B7 complexes were also tested (results provided in supplementary information, Additional file 4: Figure S1 and Additional file 5: Figure S2). These manipulated simulations described that the CTLA-4/antibody and CTLA-4/ B7-1 interactions are mostly sensitive to the rate constant values for multivalent association and dissociation of CTLA-4/B7-1 complex; whereas, the CTLA-4/B7-2 interactions are predominantly affected by the rate constants for association and dissociation of bivalent CTLA-4/B7-2 interactions. The perturbations in the latter parameters also exhibited small effects on CTLA-4/ antibody interactions. Nevertheless, none of the changes corresponding to CTLA-4/B7 interactions made any significant impacts on the CD28/B7 complexes, which were only sensitive to the rate constant values corresponding to their own association/dissociation. Although the model is sensitive to upto $50 \%$ variations in the selected kinetics parameters, the qualitative inference based on the original values remain the same. 


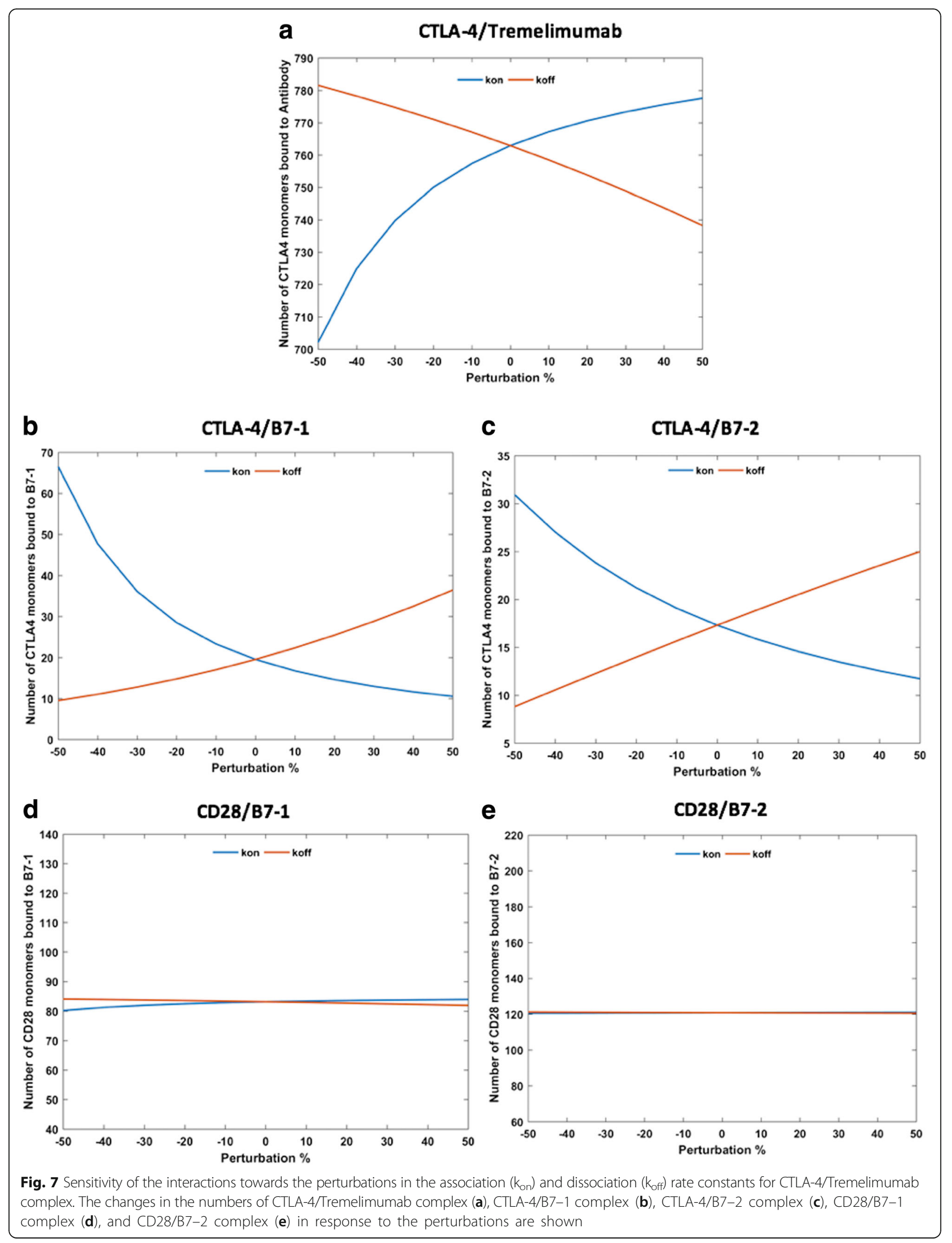




\section{Discussions}

Mathematical modeling and simulation remains a valuable tool to develop quantitative insights about the dynamic changes taking place within complex systems. It has particularly been employed in the field of cancer immunotherapy. Jansson et al. [33] developed a model for quantitative analysis of costimulatory complex formation, between CTLA4, CD28 and the B7- ligands, at the immunological synapse. However, there have been no study that modelled the effects of antibody-mediation on the complex formation at the synapse. In this study, we have taken one baby-step forward towards analyzing the effects of adding anti-CTLA-4 antibodies on the immunological balance between the co-stimulatory interactions (formed by CD28 and B7 ligands) and the co-inhibitory interactions (formed by CTLA-4 and B7 ligands) at the synapse, using a free-diffusion model. The study mainly focused on two promising CTLA-4 blocking antibodies, ipilimumab and tremelimumab, which are either in the market or in clinical trials, respectively. As acknowledged throughout the paper, this study is an extension of the Jansson's model [33], where we included several new equations and parameters to account for the effects of antibody-mediation at the synapse. The model is able to reproduce the $K_{D}$ values for the inhibition of CTLA-4 by the two antibodies. We also validated our model by showing a reasonable agreement between the dose-curves, for blocking the binding of B7-1 and B7-2 to CTLA-4 by ipilimumab, from our simulations against a previous experimental data from competitive binding assays by Keler et al. [50]. The study also helped to understand the relative efficacy of the two antibodies in CTLA-4 blockade. Although both tremelimumab and ipilimumab have similar $K_{D}$ values, the former tends to show more effective inhibition of CTLA-4/B7 interactions, due to its much lower dissociation rate that that of ipilimumab. The modelling and simulations in this work have shown that different factors, such as multivalent interactions, mobility of molecules and competition effects, could impact the effects of antibody-mediation. The results, in particular, highlighted that the competitive effects played an important role in the dose-dependent inhibition of the B7 ligand interactions with CTLA-4 receptor by the antibodies. However, it is important to concede that, as in any case of mathematical modelling, the model in this work is also mainly dependent on the parameters employed to build it. However, it is known that the $K_{D}$ values measured for the same systems under different experimental conditions could vary significantly. For example, different $k_{\text {on }}$ and $k_{\text {off }}$ rates for ipilimumab/ CTLA-4 complex have been reported in the literature. Hence, in order to minimize the impacts from such variabilities, we have used the $k_{\text {on }}$ and $k_{\text {off }}$ rates for both
ipilimumab/CTLA-4 and tremelimumab/CTLA-4 complexes from the same work of $\mathrm{He}$ et al. [1], which was very recently published.

Another important limitation of this model is that it is constructed based on the normal T-cell conditions, where both the co-stimulatory and co-inhibitory interactions at the synapse play important role in maintaining the much-needed immunological balance. However, a CTLA-4 blocking antibody (or any immune-checkpoint drug for that matter) is only administered in an abnormal micro-tumor environment, where the expression of the receptors and ligands will be different than those seen in the normal T-cells, thus shifting the balance more towards inhibitory interactions. But, unfortunately, comprehensive parameter data for simulating cancerous cells in the context of immunological synapse is not available in the literature. Hence, we made an informed choice of simulating the effects of antibody mediation in a normal T-cell environment, for which parameters are available and a preliminary model [33] (without antibody) was also published. Precisely, for this reason, we did not perform simulations by introducing the antibodies at various timescale (after reaching steady-state for instance). Instead, we only focused on simulating the competitive binding aspects of the antibodies to CTLA4 and how it changes the co-stimulatory (CD28/B7) and the co-inhibitory (CTLA-4/B7) complex formations at the synapse, when compared to untreated (or antibody-free) simulation.

Despite the stated limitations, the numerical simulations performed with the current model are in agreement with different experiments, such as the dose curve for ipilimumab-mediated inhibition of B7 ligands. The model is able to predict the dosedependent inhibition of CTLA-4/B7 interactions in an immunologically-relevant competitive environment, where both the B7-ligands and antibodies compete to bind with CTLA-4. In general, it is difficult (and not always practical) to measure the specific inhibition percentage of either B7-1 or B7-2 by the antibodies under such fully immunologically-relevant competitive binding environment. Most experiments measure the competitive binding of the anti-CTLA-4 antibodies only in the presence of either of the ligands and CTLA-4. Thus, this mathematical model could be a useful tool to gain some insights about the potencies of the antibodies to compete with both B7-1 and B7-2 to bind with the CTLA-4 receptor, at the dynamic immunological synapse. Although the simulations in this work were performed for only the two known antibodies, the model itself could serve as an easily transferable tool to study the effects of any anti-CTLA-4 antibodies on the co-stimulation by the CD28 pathway, provided the binding kinetics data for 
the query antibodies and CTLA-4 are available. Therefore, the results presented and the mathematical model will be useful for the research activity in the field of immune-checkpoints-targeted cancer therapy.

\section{Conclusion}

In this work, we have developed an expanded mathematical modeling framework to quantitatively analyze the effects of anti-CTLA-4 antibody-mediation on the costimulatory and co-inhibitory complex formation at the immunological synapse. The numerical simulations performed using this model have been validated by different experimental data. The model predicted the dose curve for the B7-ligand blockade by ipilimumab, which was in a reasonable agreement with the experimental data obtained from competitive binding assays. Further, the model was also able to reproduce the $\mathrm{K}_{\mathrm{D}}$ values for the binding of the antibodies against the CTLA-4 receptor. Our findings show that a number of significant factors, such as multivalent interactions, mobility of moleculesand competition effects contribute to the antibody-mediated interactions at the synapse. In particular, the competitive effects play a more predominant role. The simulations from our model show that in a less-competitive setting, the CTLA-4/B7-2 interactions are inhibited with much lower concentrations of antibodies, while the inhibition of B7-1 interactions required comparatively higher dose of antibodies. This is in concurrent with the previous findings that B7-1 is a preferred ligand for CTLA- 4 and also has a higher affinity to CTLA-4 compared with B7-2. Nevertheless, our simulations show that the trend is reversed within a fully competitive and dynamic immunological synapse. In fact, the antibodies are more proactive in inhibiting the divalent CTLA-4/B7-1 interactions, which in turn relieves the suppression of CTLA-4/B7-2 complexes. As a result, the inhibition of CTLA-4/B7-2 in the full model required much higher concentrations of antibodies. Further, the inhibition of the CTLA-4/B7 interactions does not essentially lead to significant increase in the costimulatory CD28/B7 complexes. It is important to acknowledge that the model suffers from some of the important limitations, which are mainly caused due to lack of several parameters required to model a tumor microenvironment. Nevertheless, the current work represents an important first step towards understanding the antibody-mediated effects on synaptic complex formation. The model could also serve as an easily transferable predictive tool to study the effects of any anti-CTLA- 4 antibodies on the co-stimulation by the $\mathrm{CD} 28$ pathway, provided the binding kinetics data for the query antibodies and CTLA- 4 are available. Our natural next step will be expanding the current model by integrating it with the simulation of the main first signal (from TCR-MHC interactions) and also connecting to some downstream signaling processes, such as interleukin-2 activation pathway. Such an integrated mathematical model will be an excellent tool to guide immune-checkpoints research towards complete elimination of cancers.

\section{Additional files}

\begin{abstract}
Additional file 1: Table S1. Parameters used in the model. The parameters related to the interactions of CTLA-4 and CD28 with the B7 ligands in the current model are provided in this file. (DOCX $26 \mathrm{~kb}$ )

Additional file 2: Table S2. Equations related to the rate of change of density for the antibody included in different complexes. The rate of change of density for the different antibody-mediated complexes are given in this file. Equations related to the rate of change of density for the antibody included in different complexes. The rate of change of density for the different antibody-mediated complexes are given in this file. (DOCX 144 kb)
\end{abstract}

Additional file 3: Table S3. Different components/species included in the model and their respective abbreviation used in the text. The different components included in our mathematical model, along with their corresponding abbreviations, are provided in this file. Different components/species included in the model and their respective abbreviation used in the text. The different components included in our mathematical model, along with their corresponding abbreviations, are provided in this file. (DOCX $13 \mathrm{~kb}$ )

Additional file 4: Figure S1. Sensitivity of the CTLA-4/antibody ( $a, d)$ and CTLA-4/B7 (b-c, e-f) interactions towards the perturbations in the association and dissociation for the CTLA-4/B7 and the CD28/B7 complexes. This file includes various figures corresponding to the sensitivity analyses performed to study the impacts of perturbations in the association and dissociation rates for the CTLA-4/B7 and the CD28/B7 complexes. (DOCX 5236 kb)

Additional file 5: Figure S2. Sensitivity of the CD28/B7-1 and CD28/B7-2 interactions towards the perturbations in the association and dissociation for the CTLA-4/B7 and the CD28/B7 complexes. This file includes various figures corresponding to the sensitivity analyses performed to study the impacts of perturbations in the association and dissociation rates for the CTLA-4/B7 and the CD28/B7 complexes. (DOCX $181 \mathrm{~kb}$ )

\section{Abbreviations}

APCs: Antigen-presenting cells; CTLA-4: Cytotoxic T-lymphocyte antigen 4; FDA: Food and drug administration; mAbs: Monoclonal antibodies; MHC: Major histocompatibility complex; ODEs: Ordinary differential equations; TCRs: T-cell receptors

\section{Funding}

The authors would like to acknowledge the funding support from Alberta Cancer Foundation (RES0025662) and Li Ka Shing Applied Virology Institute (Project ID:RES0028141) at the University of Alberta. The funding bodies had no role in this study in any way, including the study design, data collection and analysis, decision to publish and preparation of the manuscript.

\section{Availability of data and materials}

All the equations and parameter data employed in this study are provided as supplementary information. And the complete MATLAB code for the present model can be accessed by contacting the corresponding author of this work.

\section{Authors' contributions}

$A G$ and KB conceived and supervised the project. AG and TA contributed equally in relevant data collection, development of the model, testing and performing simulations. AG, TA, TC and KB analysed of the results and presentation. AG and TA wrote the manuscript. All authors read and approved the final manuscript.

Ethics approval and consent to participate Not applicable. 


\section{Competing interests}

The author declared that they have no competing interests.

\section{Publisher's Note}

Springer Nature remains neutral with regard to jurisdictional claims in published maps and institutional affiliations.

\section{Author details}

'Faculty of Pharmacy and Pharmaceutical Sciences, University of Alberta, Edmonton, Canada. ${ }^{2}$ School of Life Sciences, University of Hyderabad, Hyderabad, India. ${ }^{3}$ Department of Mathematical and Statistical Sciences, Faculty of Science, University of Alberta, Edmonton, Canada. ${ }^{4}$ Li Ka Shing Institute of Virology, University of Alberta, Edmonton, Canada.

\section{Received: 20 November 2017 Accepted: 27 April 2018 Published online: 11 June 2018}

\section{References}

1. He M, Chai Y, Qi J, Zhang CWH, Tong Z, Shi Y, Yan J, Tan S, Gao GF. Remarkably similar CTLA-4 binding properties of therapeutic ipilimumab and tremelimumab antibodies. Oncotarget. 2017

2. Ramagopal UA, Liu W, Garrett-Thomson SC, Bonanno JB, Yan Q, Srinivasan M, Wong SC, Bell A, Mankikar S, Rangan VS, et al. Structural basis for cancer immunotherapy by the first-in-class checkpoint inhibitor ipilimumab. Proc Natl Acad Sci. 2017;114(21):E4223-32.

3. Cogdill AP, Andrews MC, Wargo JA. Hallmarks of response to immune checkpoint blockade. Br J Cancer. 2017;117(1):1-7.

4. Tsai H-F, Hsu P-N. Cancer immunotherapy by targeting immune checkpoints: mechanism of T cell dysfunction in cancer immunity and new therapeutic targets. J Biomed Sci. 2017;24(1):35.

5. Lee JY, Lee HT, Shin W, Chae J, Choi J, Kim SH, Lim H, Won Heo T, Park KY Lee YJ, et al. Structural basis of checkpoint blockade by monoclonal antibodies in cancer immunotherapy. Nat Commun. 2016;7:13354.

6. Pardoll DM. The blockade of immune checkpoints in cancer immunotherapy. Nat Rev Cancer. 2012;12(4):252-64.

7. Strutt TM, McKinstry KK, Swain SL. Control of innate immunity by memory CD4 T cells. Adv Exp Med Biol. 2011:780:57-68.

8. Sharpe AH, Freeman GJ. The B7-CD28 superfamily. Nat Rev Immunol. 2002 2(2):116-26.

9. Bretscher PA. A two-step, two-signal model for the primary activation of precursor helper T cells. Proc Natl Acad Sci. 1999:96(1):185-90.

10. Bretscher P, Cohn M. A theory of self-nonself discrimination. Science (New York, NY). 1970;169(3950):1042-9.

11. Gao GF, Jakobsen BK. Molecular interactions of coreceptor CD8 and MHC class I: the molecular basis for functional coordination with the T-cell receptor. Immunol Today. 2000:21(12):630-6.

12. Gao GF, Rao Z, Bell J. Molecular coordination of a $\beta$ T-cell receptors and coreceptors CD8 and CD4 in their recognition of peptide-MHC ligands. Trends Immunol. 2002;23(8):408-13.

13. Postow MA, Callahan MK, Wolchok JD. Immune checkpoint blockade in Cancer therapy. Journal of clinical oncology : official journal of the American Society of Clinical Oncology. 2015;33(17):1974-82.

14. Grosso JF, Jure-Kunkel MN. CTLA-4 blockade in tumor models: an overview of preclinical and translational research. Cancer Immun. 2013;13:5.

15. Linsley PS, Nadler SG, Bajorath J, Peach R, Leung HT, Rogers J, Bradshaw J, Stebbins M, Leytze G, Brady W, et al. Binding stoichiometry of the cytotoxic T lymphocyte-associated Molecule-4 (CTLA-4): a DISULFIDE-LINKED HOMODIMER BINDS TWO CD86 MOLECULES. J Biol Chem. 1995;270(25): $15417-24$

16. Stamper CC, Zhang Y, Tobin JF, Erbe DV, Ikemizu S, Davis SJ, Stahl ML, Seehra J, Somers WS, Mosyak L. Crystal structure of the B7-1/CTLA-4 complex that inhibits human immune responses. Nature. 2001;410(6828):608-11.

17. Sansom DM. CD28, CTLA-4 and their ligands: who does what and to whom? Immunology. 2000;101(2):169-77.

18. Merelli B, Massi D, Cattaneo L, Mandalà M. Targeting the PD1/PD-L1 axis in melanoma: biological rationale, clinical challenges and opportunities. Crit Rev Oncol Hematol. 2014;89(1):140-65.

19. Intlekofer AM, Thompson CB. At the bench: preclinical rationale for CTLA-4 and PD-1 blockade as cancer immunotherapy. J Leukoc Biol. 2013;94(1):25-39.

20. Roh W, Chen P-L, Reuben A, Spencer CN, Prieto PA, Miller JP, Gopalakrishnan V, Wang F, Cooper ZA, Reddy SM, et al. Integrated molecular analysis of tumor biopsies on sequential CTLA-4 and PD-1 blockade reveals markers of response and resistance. Sci Transl Med. 2017:9:379.

21. Pitt Jonathan $M$, Vétizou $M$, Daillère $R$, Roberti María $P$, Yamazaki $T$, Routy $B$, Lepage P, Boneca Ivo G, Chamaillard M, Kroemer G, et al. Resistance mechanisms to immune-checkpoint blockade in Cancer: tumor-intrinsic and -extrinsic factors. Immunity. 44(6):1255-69.

22. Zaretsky JM, Garcia-Diaz A, Shin DS, Escuin-Ordinas H, Hugo W, HuLieskovan S, Torrejon DY, Abril-Rodriguez G, Sandoval S, Barthly L, et al. Mutations associated with acquired resistance to PD-1 blockade in melanoma. N Engl J Med. 2016;375(9):819-29.

23. Hodi FS, O'Day SJ, McDermott DF, Weber RW, Sosman JA, Haanen JB, Gonzalez R, Robert C, Schadendorf D, Hassel JC, et al. Improved survival with Ipilimumab in patients with metastatic melanoma. N Engl J Med. 2010; 363(8):711-23

24. Robert C, Schachter J, Long GV, Arance A, Grob JJ, Mortier L, Daud A Carlino MS, McNeil C, Lotem M, et al. Pembrolizumab versus Ipilimumab in advanced melanoma. N Engl J Med. 2015:372(26):2521-32.

25. Larkin J, Chiarion-Sileni V, Gonzalez R, Grob JJ, Cowey CL, Lao CD, Schadendorf D, Dummer R, Smylie M, Rutkowski $P$, et al. Combined Nivolumab and Ipilimumab or monotherapy in untreated melanoma. N Engl J Med. 2015;373(1):23-34.

26. Wolchok JD, Kluger H, Callahan MK, Postow MA, Rizvi NA, Lesokhin AM, Segal NH, Ariyan CE, Gordon R-A, Reed K, et al. Nivolumab plus Ipilimumab in advanced melanoma. N Engl J Med. 2013;369(2):122-33.

27. Postow MA, Chesney J, Pavlick AC, Robert C, Grossmann K, McDermott D, Linette GP, Meyer N, Giguere JK, Agarwala SS, et al. Nivolumab and Ipilimumab versus Ipilimumab in untreated melanoma. N Engl J Med. 2015; 372(21):2006-17.

28. Comin-Anduix B, Escuin-Ordinas H, Ibarrondo FJ. Tremelimumab: research and clinical development. OncoTargets and therapy. 2016;9:1767-76

29. Kaplon A, Reichert JM. Antibodies to watch in 2018. mAbs. 2018:10(2): 183-203.

30. Linsley PS, Brady W, Urnes M, Grosmaire LS, Damle NK, Ledbetter JA. CTLA-4 is a second receptor for the B cell activation antigen B7. J Exp Med. 1991; 174(3):561-9.

31. van der Merwe PA, Bodian DL, Daenke S, Linsley P, Davis SJ. CD80 (B7-1) binds both CD28 and CTLA-4 with a low affinity and very fast kinetics. J Exp Med. 1997;185(3):393-404

32. Sengers BG, McGinty S, Nouri FZ, Argungu M, Hawkins E, Hadji A, Weber A, Taylor A, Sepp A. Modeling bispecific monoclonal antibody interaction with two cell membrane targets indicates the importance of surface diffusion. MAbs. 2016:8(5):905-15.

33. Jansson A, Barnes E, Klenerman P, Harlén M, Sørensen P, Davis SJ, Nilsson P. A theoretical framework for quantitative analysis of the molecular basis of Costimulation. J Immunol. 2005;175(3):1575.

34. Aravindhan G, Kumar GR, Kumar RS, Subha K. AJAX Interface: a breakthrough in bioinformatics web applications. Proteomics Insights. 2009; 2(PRI-2-Ganesan-et-al):1-7.

35. Aravindhan G, Kumar RS, Subha K, Subazini TK, Dey A, Kant K, Kumar GR. AIM-BLAST-AJAX interfaced multisequence Blast. Proteomics Insights. 2009; 2(PRI-2-Ganesan-et-al-2):9-13.

36. Subha K, Kumar GR, Rajalakshmi R, Aravindhan G. A novel strategy for mechanism based computational drug discovery. Biomarkers in Cancer. 2010;2:35-42.

37. Kronik N, Kogan Y, Schlegel PG, Wölfl M. Improving T-cell immunotherapy for melanoma through a mathematically motivated strategy: efficacy in numbers? J Immunother. 2012;35(2):116-24.

38. Kirschner D, Panetta JC. Modeling immunotherapy of the tumor - immune interaction. J Math Biol. 1998:37(3):235-52.

39. Sanga $S$, Sinek JP, Frieboes HB, Ferrari M, Fruehauf JP, Cristini V Mathematical modeling of cancer progression and response to chemotherapy. Expert Rev Anticancer Ther. 2006;6(10):1361-76.

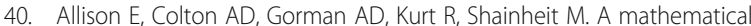
model of the effector cell response to cancer. Math Comput Model. 2004; 39(11):1313-27.

41. Robertson-Tessi M, El-Kareh A, Goriely A. A mathematical model of tumorimmune interactions. J Theor Biol. 2012;294:56-73.

42. de Pillis LG, Radunskaya AE, Wiseman CL. A validated mathematical mode of cell-mediated immune response to tumor growth. Cancer Res. 2005; 65(17):7950 
43. Bidot C, Gruy F, Haudin CS, El Hentati F, Guy B, Lambert C. Mathematical modeling of T-cell activation kinetic. J Comput Biol: a journal of computational molecular cell biology. 2008;15(1):105-28.

44. Cheng X, Veverka V, Radhakrishnan A, Waters LC, Muskett FW, Morgan S, Huo J, Yu C, Evans EJ, Leslie AJ, et al. Structure and interactions of the human programmed cell death 1 receptor. J Biol Chem. 2013;288(17): $11771-85$.

45. Egen JG, Allison JP. Cytotoxic T lymphocyte Antigen-4 accumulation in the immunological synapse is regulated by TCR signal strength. Immunity. 2002;16(1):23-35.

46. Chikuma S. CTLA-4, an essential immune-checkpoint for T-cell activation. In: Yoshimura A. (eds) Emerging concepts targeting immune checkpoints in cancer and autoimmunity. Current Topics in Microbiology and Immunology. Cham:: Sprinter. 2017:410.

47. Bell GI. Models for the specific adhesion of cells to cells. Science (New York, NY). 1978;200(4342):618.

48. Collins AV, Brodie DW, Gilbert RJC, laboni A, Manso-Sancho R, Walse B, Stuart DI, van der Merwe PA, Davis SJ. The interaction properties of costimulatory molecules revisited. Immunity. 2002;17(2):201-10.

49. Pentcheva-Hoang T, Egen JG, Wojnoonski K, Allison JP. B7-1 and B7-2 selectively recruit CTLA-4 and CD28 to the immunological synapse. Immunity. 2004;21(3):401-13.

50. Keler T, Halk E, Vitale L, O'Neill T, Blanset D, Lee S, Srinivasan M, Graziano RF, Davis T, Lonberg N, et al. Activity and safety of CTLA-4 blockade combined with vaccines in Cynomolgus macaques. J Immunol. 2003;171(11):6251-9.

51. Greene JL, Leytze GM, Emswiler J, Peach R, Bajorath J, Cosand W, Linsley PS Covalent dimerization of CD28/CTLA-4 and oligomerization of CD80/CD86 regulate T cell costimulatory interactions. J Biol Chem. 1996;271(43):26762-71.

\section{Ready to submit your research? Choose BMC and benefit from:}

- fast, convenient online submission

- thorough peer review by experienced researchers in your field

- rapid publication on acceptance

- support for research data, including large and complex data types

- gold Open Access which fosters wider collaboration and increased citations

- maximum visibility for your research: over $100 \mathrm{M}$ website views per year

At BMC, research is always in progress.

Learn more biomedcentral.com/submissions 